\title{
High Performance Multi-Vendor Network Data Acquisition Platform
}

\author{
Domingos Henrique Beolchi Rios \\ EMBRAER S.A. - Gavião Peixoto - SP - Brazil, \\ domingos.rios@embraer.com.br
}

\begin{abstract}
:
As the aircrafts are becoming more complex, the amount of data to be captured and processed during the test flights increases a lot and that is what we saw in the past few years. This is a result of the increment on the number and diversity of aircraft buses and the need for more complex acquisition systems to face the requirements and the demand from engineering. This paper describes the architecture of one system that not only met these requirements but introduce new concepts such as data redundancy and multivendor support. The Acquisition System is $100 \%$ Network Based but also has one PCM output that is used during Telemetry. This paper also brings some solutions to problems like the high network bandwidth on board, the amount of data recorded during the flights and the manipulation of it on the ground after the flight to allow efficient post flight analysis. There are also additional challenges with the integration of equipment from multiple vendors and the real-time visualization both onboard and during Telemetry.
\end{abstract}

Key words: Network Data Acquisition, PCM, Real Time, Data Processing, Flight Test.

\section{Introduction}

We have being using PCM based acquisition systems from several years in many different aircrafts. Actually, we still have some of them operating and producing good results and data to be analyzed. The problem was that on some of the projects we had do acquire a large number of parameters (and consequently data) that has to be processed and stored during the flights. In order to properly handle all the requirements passed to us we decided to use an Ethernet base architecture, much more flexible and capable of accommodate many different data sources and large amount of data. We did this on a smooth way using the first project as a transition (PCM based with some Ethernet data) and finally got the full Ethernet on the second one (Ethernet Based with some PCM) [1]
Since this second one was bigger than the previous, we also took advantage from the weight savings on cable harness and the extra flexibility to accommodate analog data and many aircraft buses on the same system.

During the design and integration we faced several challenges, some of them are shared here.

\section{Ehternet Based does not mean no PCM}

We have a small PCM stream that is used only during Telemetry Sessions. It has less than 3\% of all the data acquired and most of the parameters are under sampled on that transmission. This is another benefit of Ethernet acquisition: we can have the same data sampled with different rates without compromising the post flight analysis. This PCM stream has no use for us after the flight and is discarded. 


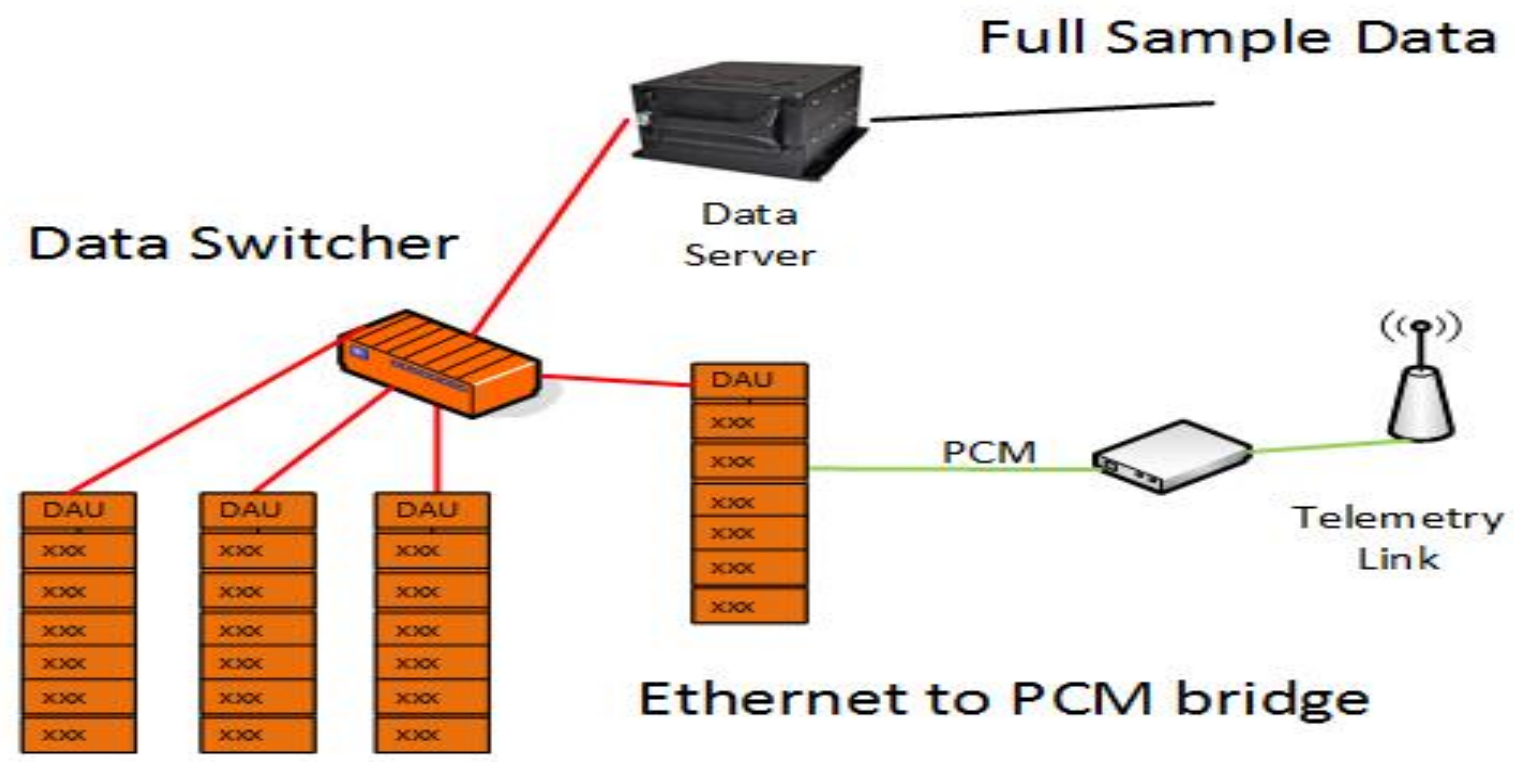

\section{DAUs}

Fig. 1. PCM for Telemetry.

\section{High Network Bandwidth}

Ethernet is non-deterministic so we had to count on good acquisition timestamping, network traffic stability, zero packet loses and spare bandwidth to have a reliable system.

Added to this was the large number of parameters and aircraft buses required that caused high data rates on the acquisition network. To handle this we decided to physically segregate the data acquisition network from the other aircraft network traffic used during the flight (typically compressed video and Engineering Workstations). By doing this we end up with a network with relatively stable traffic during all the flight and spare bandwidth. The time synchronization was done by the standard IEEE-1588.

Another challenge derived from the high bandwidth was to find good Ethernet switchers because they are the heart of Ethernet based Acquisition Systems. The switchers must not only synchronize the time of all the acquisition units but also deliver every single package produced by them to the recorders and Data Servers on board with zero loses. Since the Data Acquisition Units are spread along the aircraft, we had to install many switchers on board distributed on layers to aggregate the traffic towards the recorders and servers. 


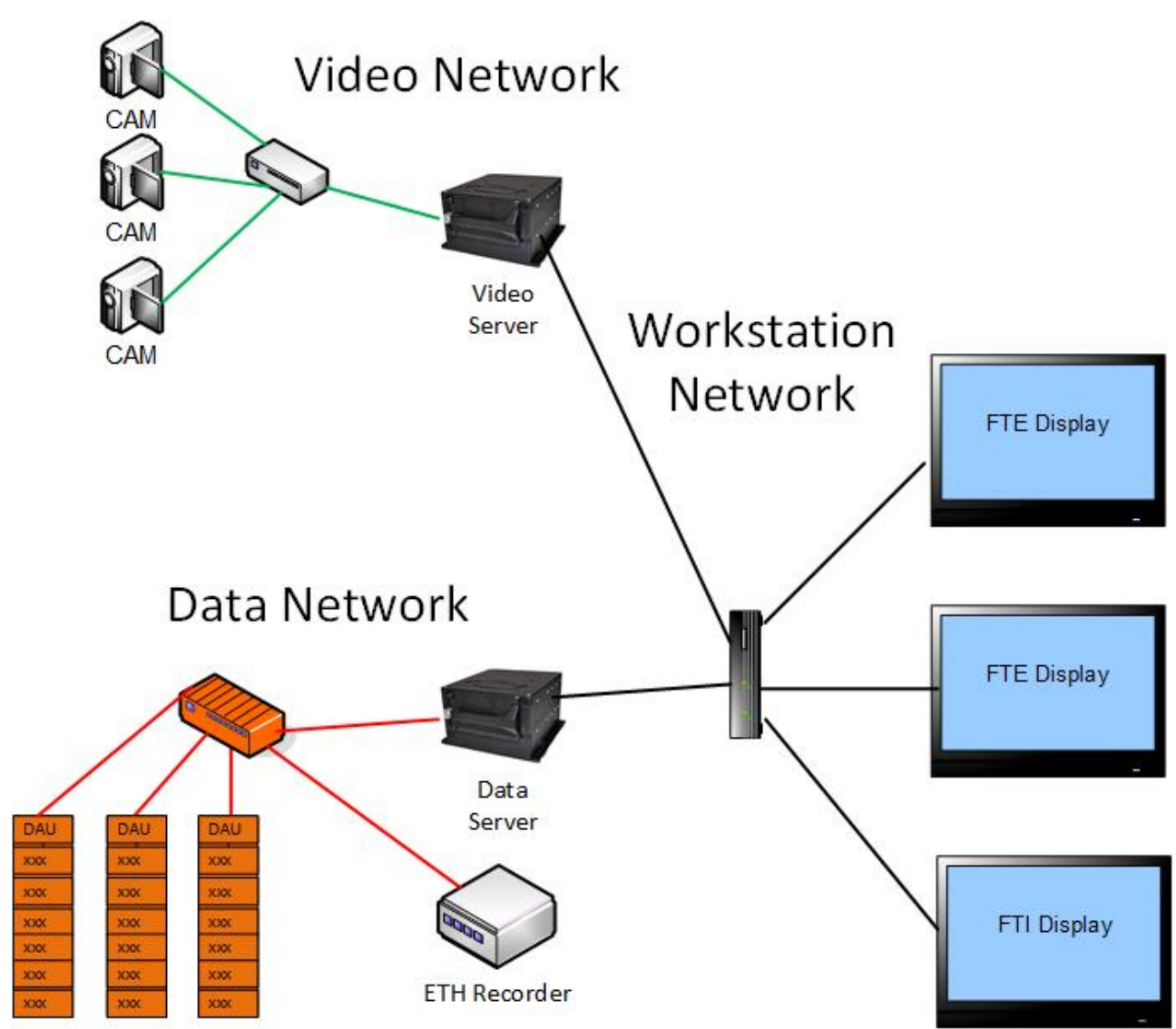

Fig. 2. Segregated Network Configuration.

All the servers are also recorders so they have removable SSD modules to be collected after the flights. In order to have a Dissimilarity we

\section{Fault Tolerant}

To improve the system reliability we designed a dual redundant network for acquisition and processing. If we have a failure on switcher, cable or server we don't lose the flight. Since the video acquisition was not essential on most of the flights we only have dual video servers an not the full network infrastructure as we have on Data Acquisition. added a third recorder that receives data from all the primary networks and just record them as a backup (no processing).

We also had to include a Redundancy removal layer on the Visualization Platform so it is transparent to the Engineer on board the source that is being used to receive the data. 


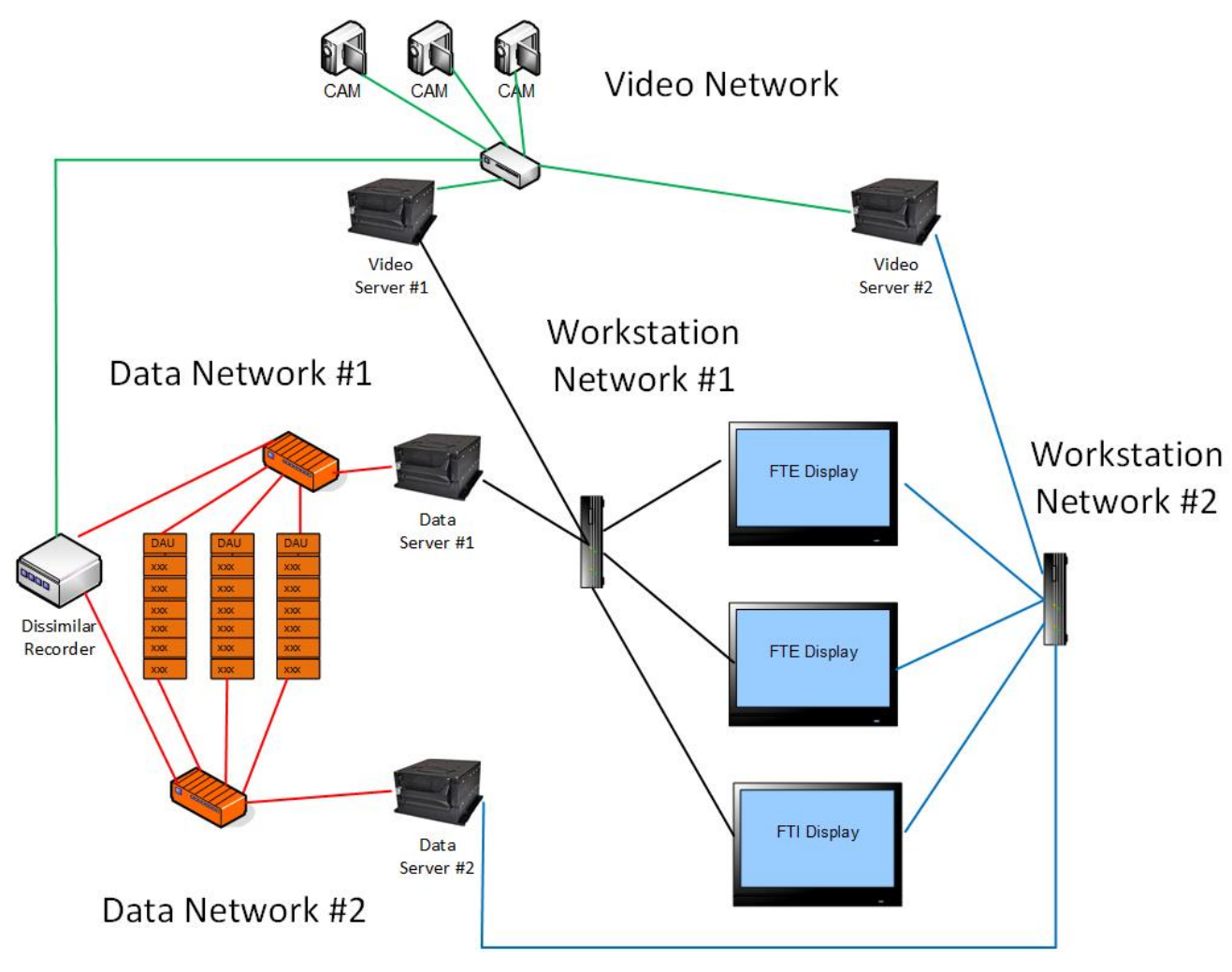

Fig. 3. Redundant Networks.

\section{Amount of Data}

With all the bandwidth and the redundancy installed, we end up with a huge amount of data being recorded after each flight. Even a single recording can have 1 Terabyte of information after certain test flights. To reduce the time spend to made the data available to be analyzed by the Engineering we have designed a way to not copy any data to fulfill the first requests just after the flight. To do this we insert the very same SSD module used on board into the Servers on the ground station. The actual copy and backup processes are done hours after the flight when the servers are not being used. Each flight uses 5 or 6 SSD modules, depending on the configuration.

We also have High Speed SSD modules with multiple access lines that allow us to read at 2 GBytes/s. To reduce the amount of data recorded for historical purposes we store only the Raw Data (zero processed data stored) by using an on-demand data processing configuration. 


\section{High Performance SSD Flight Data Servers}

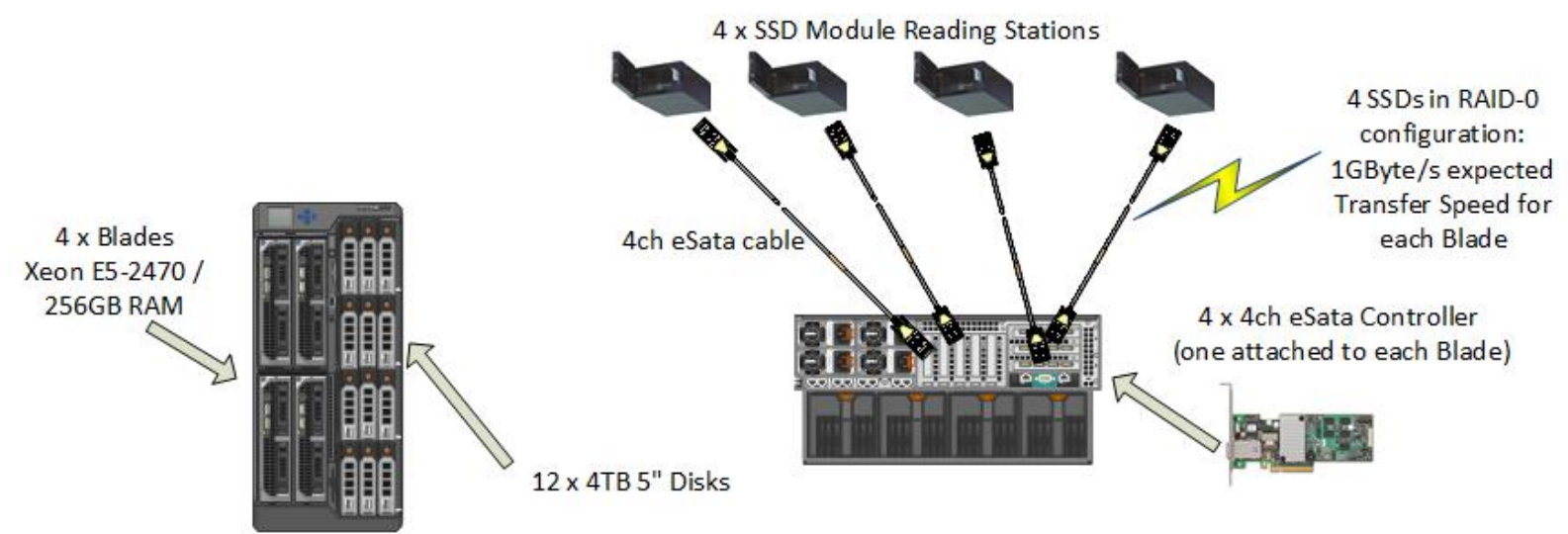

Fig. 4. Flight Data Servers.

\section{Multiple Vendors}

Not a single manufacturer had a solution to all of our requirements of data acquisition (like high data rates, optimized bus acquisition, recorders, switchers, etc.). So we had to design a system capable of receive data from multiple manufacturers and with different hardware platform. Everything must be converted to
Ethernet first (usually with distinct protocols). After that, we collect all the sources in the main switcher and send the packages for recording and processing.

We decided to use a single protocol for the processed data (after RAW to EU conversion) and we had to write specific modules on the server to handle each different input protocol and convert the data to the same output format to be sent over the Workstation Network.

Integrate such a system is another big challenge because of the diversity of hardware, configuration programs and usually some particular view of the International Standards.

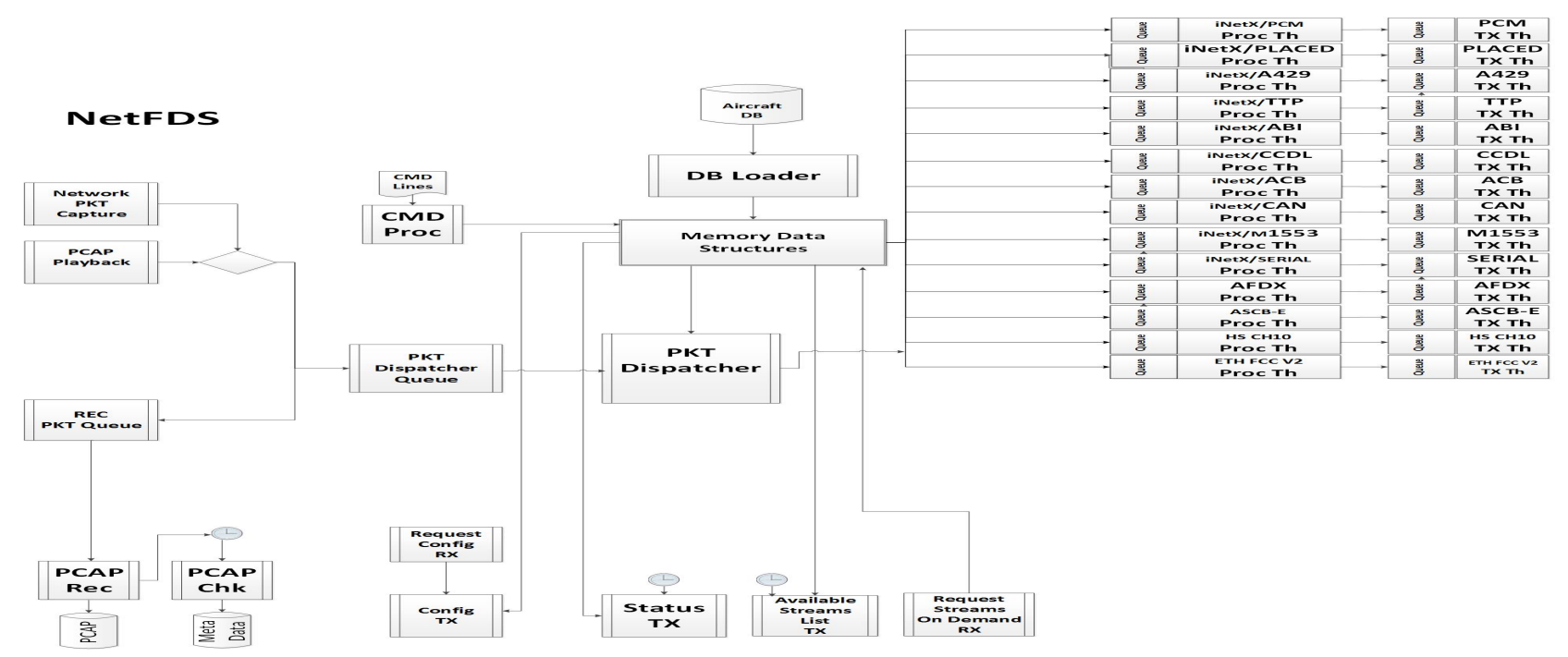

Fig. 5. Main Data Processor Software architecture. 


\section{Visualization Platform}

The requirements for the Visualization Platform were not simple and due to the numbers involved, the job was not an easy one. The initial requirements include:

- High number of data sources (500+);

- High number of parameters $(100 \mathrm{~K}+)$;

- Ethernet bandwidth (300Mbps+);

- Redundancy (2 networks).

- Single platform onboard and during Telemetry sessions;

Custom displays (pilot, PIO)

Needless to say that we did not find a comercial tool that has all those requirements.

So we end up developing our own platform and we had to start from scratch because our previous versions were just for PCM systems.
- Electronic Flight Card Integration (Test Points, Procedures, Load Configuration changes, refueling);

- True Real-time display (all samples with coherency);

- Distinct time stamps for each data source;

- Integration with analysis tools (spreadsheets, Mathlab, $3^{\text {rd }}$ party)

The software uses a common client interface that handles all network protocol and put the data in a shared memory to be used by client applications. The data can be also shared among other workstations over the network.

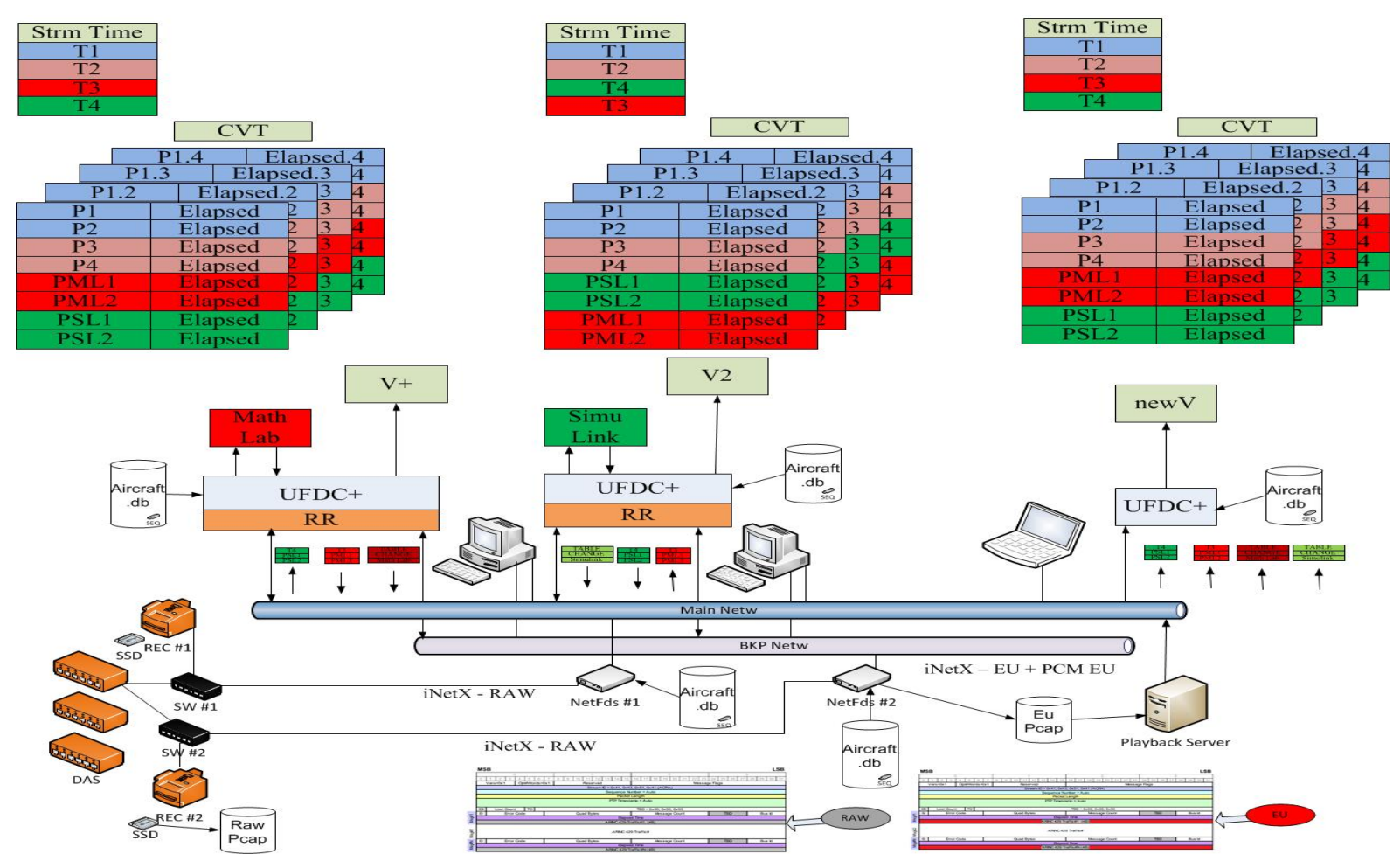

Fig. 6. Main Data Processor Software architecture. 


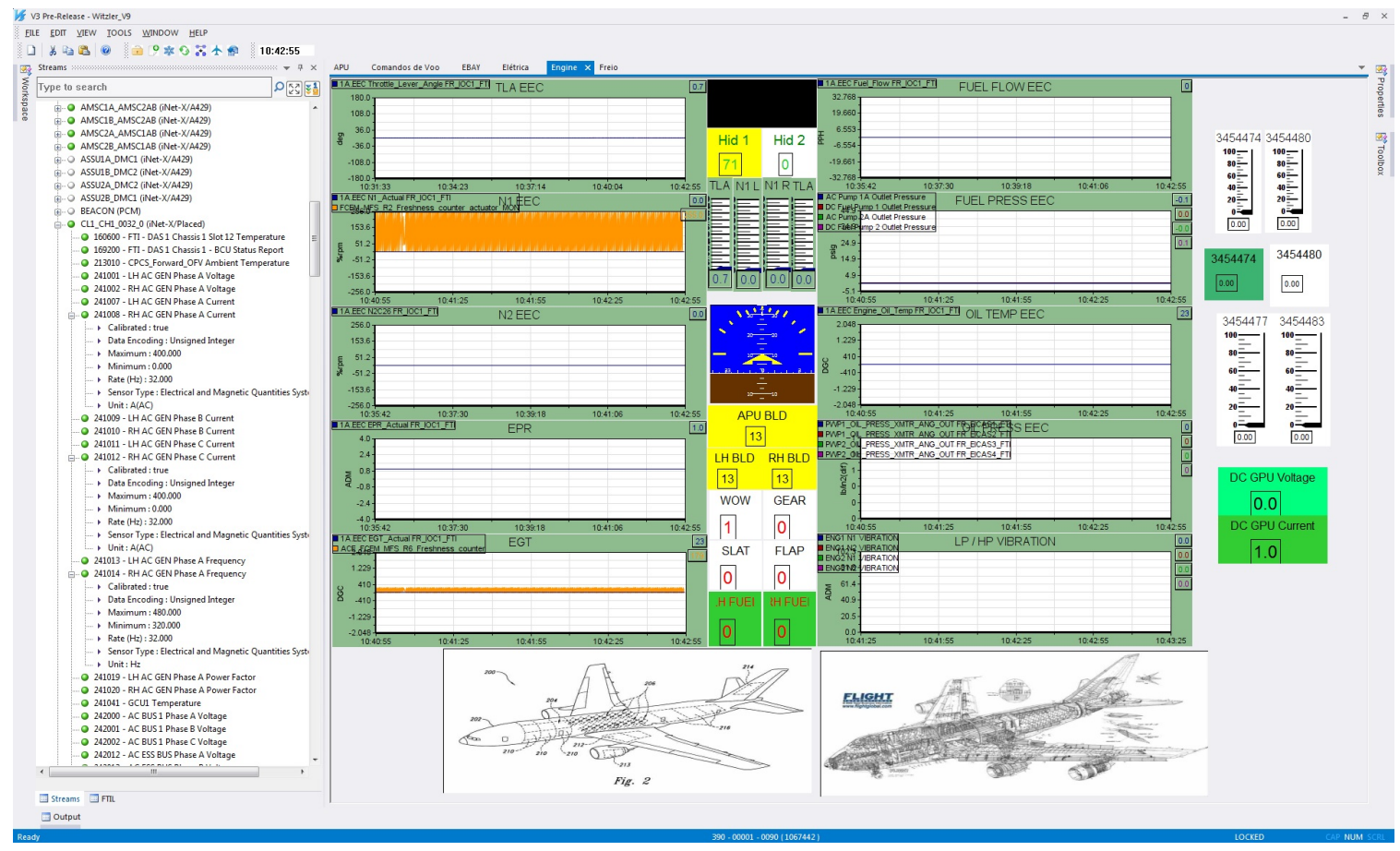

Fig. 7. Screen Sample of the Visualization Platform.

\section{Conclusion}

The System explained here is on its second generation so it has been improved (and

increased) from its original configuration. It is being used by many aircrafts with very good results.

\section{References}

[1] Luiz Fernando de Souza, Domingos

Henrique Beolchi Rios and Stephen Willis, 2012, "PCM to Ethernet: A Hybrid System Used to Certify the Next Generation of Data Transfer Technology", Proceedings of the International Telemetry Conference 2012 